\title{
EIGENSOLUTION OF NONCLASSICALLY DAMPED ASYMMETRIC VIBRATION SYSTEMS
}

\author{
ElSayed M. ElBeheiry \\ Department of Production Engineering and Mechanical design, \\ Faculty of Engineering, Menoufia University, \\ Shebin ElKom, Egypt
}

\begin{abstract}
This paper presents a new effective approach to solving the generalized eigenvalue problem $B \mathbf{u}=\lambda \boldsymbol{A} \mathbf{u}$ by using a perturbation technique." "A generalized second-order perturbation theory is adapted such that the perturbed solution will be based on the eigensolution of an unperturbed subproblem describing the conservative part of the original nonclassically, viscously damped system with asymmetric mass, damping and stiffness matrices. Two numerical examples in addition to a practical problem are studied to show that the eigensolutions obtained by the current method match, to a great extent, solutions obtained by time-consuming exact methods.
\end{abstract}

\section{KEY WORDS}

Eigenproblems, asymmetric systems, nonproportional damping, perturbation.

\section{INTRODUCTION}

The eigenvalue problem is the heart of the linear vibration theory, and its solution provides the vibration analyst with rich foundation about the behavior of his system from the stability and response points of view. This is the reason of the eigenvalue problem being always under focus of continuous, intensive research activity everywhere. In the absence of dissipative forces, generally, the linear dynamic systems possess classical normal modes [1]. In other words, they have a complete set of real orthogonal eigenvectors that can transform the system into a diagonal form. This form is very delicate for applying the powerful modal superposition method to response calculations. So many structure problems are lightly damped, and can be assumed to have symmetric damping matrix that is proportional to symmetric mass and stiffness matrices.

Manuscript received from Dr .Elsayed $M$. Eibeheiry

Accepted on : 30/1/2001

Engineering Research Journal Vol 24,No 2, 2001 Minufiya University, Faculty Of

Engineering , Shebien El-Kom , Egypt, ISSN . 1110-1180 
The self-adjoint eigensolution is then an easy task to achieve by powerful tools [2] because the system classical normal modes are conserved for proportionally damped systems. These tools become unacceptable even for lightly damped systems having symmetric viscous damping matrices of distribution dissimilar to that of symmetric mass and stiffness matrices. The system is then called nonclassically damped, and response predictions urge using other techniques. A common procedure in the analysis of such systems is to neglect the off-diagonal elements of the associated modal damping matrix. Some other methods for modal and response calculations of nonclassically damped are available in the literature [3-6].

In modern vibration practices [7,8], active damping and fully active vibration control techniques, normally, lead to asymmetric damping and stiffness matrices. Moreover, introducing circulatory forces and gyroscopic moments can further complicate the eigenvalue problem, as it becomes quadratic and asymmetric one. This necessitates the use of other methods like the pioneering Duncan's formulation [9] in which the concept of trivial identity was introduced by Duncan to linearize the problem. But, before going to algorithms that counts on Duncan's formulation or any other methods as in references [1013], asymmetric systems might possess classical normal modes, and must be checked for their existence. Thus one can avoid complexity in computations and consumption in time, especially, in large-scale models. Conditions under which classical normal modes exist in asymmetric systems are presented in references [14-16].

First- and second-order perturbation techniques have been proven effective in both eigensolution calculations and eigensolution reanalysis problems [17-20]. Meirovitch and Ryland [21] made a second-order perturbation theory developed for the generalized eigensystem $\lambda \mathbf{u}=\boldsymbol{A u}$, fruitful for application to lightly damped gyroscopic systems with symmetric mass, damping and stiffness matrices. Chung and Lee [22] extended the theory for application to the generalized eigenproblem $B \mathbf{u}=\lambda A \mathbf{u}$ of heavy, but weakly nonproportional damped systems. Although the matrix $\boldsymbol{A}$ in the basic perturbation theory and the matrices $\boldsymbol{A}$ and $\boldsymbol{B}$ in its extension have no restriction except that they must be real, simplifications are necessary to make this theory attractive for application to large scale systems where hundreds or thousands of degrees of freedom can be considered. Basically, the theory requires the calculation of eigenvalues and right and left eigenvectors of the unperturbed system. This will be the gate for any simplification to be significant.

This paper presents an approach by which the eigensolution by using the second-order perturbation theory when applied to asymmetric system can be based on the solution of unperturbed conservative system formulated in a highly standard eigenvalue problem of single, symmetric positive definite matrix. Numerical examples will be presented to demonstrate the method in a detailed manner. 


\section{NEW FORMULATION}

Consider the free vibration problem of a general linear discrete system described by vector differential equation

$$
M \ddot{q}(t)+(C+G) \dot{\mathbf{q}}(t)+(K+H) \mathrm{q}(t)=0
$$

where $\boldsymbol{M}, \boldsymbol{C}$, and $K$ are $n \times n$ real asymmetric matrices. $M$ is the mass matrix, $C$ is the damping matrix, $K$ is the stiffness matrix, $\boldsymbol{G}$ is an $n \times n$ real skew symmetric gyroscopic matrix, $\boldsymbol{H}$ is an $n \times n$ real skew symmetric circulatory matrix, and $\mathrm{q}(t)$ is a real $n \times 1$ vector of generalized coordinates. Note here that $\boldsymbol{G}$ is of conservative nature, while $\boldsymbol{H}$ is a dissipative one. If the trivial identity

$$
(K+H) \dot{q}-(K+H) \dot{q}=0
$$

adjoins Eq. (1), the $2 n$ associated eigenvalue problem and its adjoint will be:

$$
B \mathbf{u}_{i}=\lambda_{i} A \mathbf{u}_{i}, \quad B^{T} \mathbf{v}_{i}=\lambda_{i} A^{T} \mathbf{v}_{i}, \quad i=1,2, \ldots ., 2 n ;
$$

where $\mathbf{q}=e^{\lambda t} \mathbf{u}$ is substituted into Eq. (1) and (2) for exponential form solutions, $\lambda_{i}$ is the ith eigenvalue, $\mathfrak{u}_{i}$ and $\mathbf{v}_{i}$ are the corresponding right and left eigenvectors, respectively, of the non-self-adjoint eigenvalue problem (3). The biorthogonality of right and left eigenvectors provides

$$
\begin{gathered}
\mathbf{v}_{j}^{T} A \mathbf{u}_{i}=\mathbf{u}_{j}^{T} A \mathbf{v}_{i}=2 a_{i} \delta_{i j}, \\
\mathbf{v}_{j}^{T} B \mathbf{u}_{i}=\mathbf{u}_{j}^{T} B \mathbf{v}_{i}=2 a_{i} \lambda_{i} \delta_{i j}, \quad i, j=1,2, \ldots, 2 n
\end{gathered}
$$

where $a_{i}$ is the scale factor of the $i$ th eigenvector, $\delta_{i j}$ is the Kronecker delta. $\boldsymbol{A}$ and $\boldsymbol{B}$ are real asymmetric matrices defined by

$$
\boldsymbol{A}=\left[\begin{array}{cc}
\boldsymbol{K}+\boldsymbol{H} & 0 \\
0 & \boldsymbol{M}
\end{array}\right], \quad \boldsymbol{B}=\left[\begin{array}{cc}
0 & -\boldsymbol{K}-\boldsymbol{H} \\
\boldsymbol{K}+\boldsymbol{H} & \boldsymbol{C}+\boldsymbol{G}
\end{array}\right]
$$

Since any real asymmetric matrix can be regarded as a summation of two real matrices one of them symmetric and the other one is skew symmetric, the asymmetric matrices $M, C$ and $\boldsymbol{K}$ can be written as follows:

$$
M=M_{0}+M_{g}, \quad C=C_{0}+C_{g}, \quad K=K_{0}+K_{g}
$$


where $M_{0}, C_{0}$ and $K_{0}$ are symmetric matrices, and $M_{g}, C_{g}$ and $K_{g}$ are skew symmetric ones. For instance, the calculated symmetric and skew symmetric parts of the damping matrix are:

$$
C_{0}=\left(C+C^{T}\right) / 2, \quad C_{g}=\left(C-C^{T}\right) / 2
$$

It should be mentioned here that the skew symmetric matrix $C_{g}$ represents the conservative part of the damping matrix [23,24]. Normally, the true damping is contained into the symmetric part $C_{0}$ of the asymmetric damping matrix $C$ $[23,24]$. It will be further assumed that $M_{0}$ and $K_{0}$ are positive definite. For perturbation purposes, if Eq. (6) is substituted into Eq. (5), one can write the matrices in Eq. (5) as

$$
A=A_{0}+A_{1}, \quad B=B_{0}+B_{1}
$$

where $A_{0}$ and $B_{0}$ are considered as unperturbed matrices, and $A_{1}$ and $B_{1}$ are considesed as perturbation matrices. The matrices $A$ and $B$ are then called the perturbed matrices. An order of magnitude condition is considered here [22], which states that the elements of the matrices $A_{1}$ and $B_{1}$ are one order of magnitude smaller than the elements of $A_{0}$ and $B_{0}$. The following formulation is suggested for the matrices in Eq. (8):

$$
\begin{gathered}
A_{0}=\left[\begin{array}{cc}
K_{0} & 0 \\
0 & M_{0}
\end{array}\right], \quad B_{0}=\left[\begin{array}{cc}
0 & -K_{0} \\
K_{0} & G+C_{g}
\end{array}\right] \\
A_{1}=\left[\begin{array}{cc}
-K_{g}^{T}-H^{T} & 0 \\
0 & M_{g}
\end{array}\right], B_{1}=\left[\begin{array}{cc}
0 & K_{g}^{T}+H^{T} \\
K_{g}+H & C_{0}
\end{array}\right]
\end{gathered}
$$

where $K_{0}$ and $M_{0}$ are assumed symmetric positive definite matrices, $A_{0}$ and $B_{0}$ will be symmetric positive definite and skew symmetric, respectively. While the matrices $A_{1}$ and $B_{1}$ are skew symmetric and symmetric nonnegative definite, respectively. The reason for suggesting that new matrix formulation in Eqs. (9) and (10) is that the unperturbed and perturbation matrices are either symmetric of skew symmetric. In other words, the skew symmetric matrix $A_{1}$ is a perturbation to the symmetric matrix $A_{0}$ while the symmetric matrix $\boldsymbol{B}_{1}$ is a peturbation to the skew symmetric matrix $\boldsymbol{B}_{0}$. This permits taking advantages of this arrangement in the modified perturbation theory that will be presented later on. Note also here that the unperturbed matrices $A_{0}$ and $B_{0}$ represent the conservative gyroscopic part of the original perturbed system $\boldsymbol{A}$ and $\boldsymbol{B}$. 


\section{GENERAL PERTURBATION THEORY}

The unperturbed eigenvalue problem is assumed to have known eigensolution. In general, the accuracy of the perturbation process is pertinent to the accuracy of the unperturbed solution. The unperturbed eigenproblem and its adjoint one can be expressed as follows:

$$
B_{0} \mathbf{u}_{0 i}=\lambda_{0 i} A_{0} \mathbf{u}_{0 i}, \quad B_{0}^{T} \mathbf{v}_{0 i}=\lambda_{0 i} A_{0}^{T} \mathbf{v}_{0 i}, \quad i=1,2, \ldots ., 2 n
$$

where $\lambda_{0 i}$ is the ith eigenvalue, and $\mathbf{u}_{0 i}$ and $\mathbf{v}_{0 i}$ are iths right and left eigenvectors, respectively. The biorthogonality property of the right and left eigenvectors satisfies the following relations:

$$
\begin{gathered}
\mathbf{v}_{0 j}^{T} A_{0} \mathbf{u}_{0 i}=\mathbf{u}_{0 j}^{T} A_{0} \mathbf{v}_{0 i}=2 a_{i} \delta_{i j} \\
\mathbf{v}_{0 j}^{T} B_{0} \mathbf{u}_{0 i}=\mathbf{u}_{0 j}^{T} B_{0} \mathbf{v}_{0 i}=2 a_{i} \lambda_{0 i} \delta_{i j}, \quad i, j=1,2, \ldots, 2 n .
\end{gathered}
$$

To produce the perturbed eigenvalues in terms of the unperturbed ones, one can express the solution of the perturbed eigenvalues as follows:

$$
\begin{aligned}
\lambda_{i}=\lambda_{0 i}+\lambda_{1 i}+\lambda_{2 i}+\ldots, & i=1,2, \ldots, 2 n \\
\mathbf{u}_{i}=\mathbf{u}_{0 i}+\mathbf{u}_{1 i}+\mathbf{u}_{2 i}+\ldots, & i=1,2, \ldots, 2 n \\
\mathbf{v}_{i}=\mathbf{v}_{0 i}+\mathbf{v}_{1 i}+\mathbf{v}_{2 i}+\ldots, & i=1,2, \ldots, 2 n
\end{aligned}
$$

The order of any particular term in Eq. (13) is characterized by the first subscript. For example, $\lambda_{1 i}, \mathbf{u}_{1 i}$ and $\mathbf{v}_{1 i}$ are one order of magnitude smaller than $\lambda_{0 i}, \mathbf{u}_{0 i}$ and $\mathbf{v}_{0 i}$, respectively. Substituting Eqs. (8) and (13) into (4) gives, after collection by order of magnitude, the perturbation systems as summarized in (Eqs. (A1) : (A3)) Appendix A. The first-order perturbations $\mathbf{u}_{1}$ and $\mathbf{v}_{1}$ can be expressed as a linear combinations of $\mathbf{u}_{0}$ and $\mathbf{v}_{0}$, respectively, because they span the same space:

$$
\mathbf{u}_{1 i}=\sum_{k=1}^{2 n} \varepsilon_{i k} \mathbf{u}_{0 k}, \quad \mathbf{v}_{1 i}=\sum_{k=1}^{2 n} \gamma_{i k} \mathbf{v}_{0 k} \quad i=1,2, \ldots, 2 n
$$

where $\varepsilon_{i k}$ and $\gamma_{i k}$ are small first order coefficients. Similarly, the eigenvectors $\mathbf{u}_{2}$ and $\mathbf{v}_{2}$ can be expressed as: 


$$
\mathbf{u}_{2 i}=\sum_{k=1}^{2 n} \widetilde{\varepsilon}_{i k} \mathbf{u}_{0 k}, \quad \mathbf{v}_{2 i}=\sum_{k=1}^{2 n} \widetilde{\gamma}_{i k} \mathbf{v}_{0 k} \quad i=1,2, \ldots ., 2 n
$$

where $\widetilde{\varepsilon}_{i k}$ and $\widetilde{\gamma}_{i k}$ are small second order coefficients. The solutions for first and second order perturbation problems are summarized in Appendix A. Euations (A4) and (A5) solve for the first order perturbation problem while Eqs. (A6) : (A8) solve for the second order one.

\section{MODIFIED PERTURBATION THEORY}

The conservative gyroscopic system that is represented by the matrices $A_{0}$ and $\boldsymbol{B}_{0}$ in Eq. (9) is considered as the unperturbed system of equations. If the Qutcomes of the eigenvalue problem (11) of the unperturbed system (9) satisfy the orthogonality conditions (12), then one can say that the first- and secondolder perturbation solutions (Eqs. (A4) : (A8)) are possible. Unfortunately, the results of the second order perturbation theory derived by Chung and Lee [22], although they are quite general with a single restriction that $\boldsymbol{A}$ and $\boldsymbol{B}$ must be real, are not liable for application to the unperturbed system (11). In other worls, the solution of the unperturbed (11) with the matrices $A_{0}$ and $B_{0}$ as given in Eq. (9), violates the orthogonality arrangements as given by Eq. (12) and, consequently, mismatches the formulation requirements of the secondorder perturbation theory. The task now is to modify this theory to make it liable for application to unperturbed systems like the one considered in this study. The following theorem will clarify this issue.

Theorem I: The solution of the unperturbed eigenproblem (11), with the matrices $A_{0}$ and $B_{0}$ as defined in $E q$. (9), mismatches the formulation requirements of Eq. (12) that led to the solution results (Eqs. (A4) : (A8)) of the general perturbation theory as derived by Chung and Lee [22]. Thus, the solution of the first-and second-order perturbation problems (Eqs. (A2) and (A3)) is not possible by using Eqs. (A4) : (A8) unless $-\mathbf{u}_{0 j}$ replaces $\mathbf{v}_{0 j}$ in Eqs. (A4) and (A5) with the sign reversed at the right hand sides of Eqs. (A6) : (A8).

Proof: Since $A_{0}$ is symmetric positive definite and $B_{0}$ is skew symmetric, the eigenvalues of the unperturbed eigensystem (11) will be pure imaginary complex conjugate pairs and the eigenvectors will also be complex conjugate pairs with the following properties [21]:

$$
\begin{aligned}
& \lambda_{0_{2 r}}=\bar{\lambda}_{0_{2 r-1}}, \quad r=1,2, \ldots ., n \\
& \mathbf{u}_{0_{2 r}}=\overline{\mathbf{u}}_{0_{2 r-1}}, \quad r=1,2, \ldots . . n
\end{aligned}
$$




$$
\mathbf{v}_{0_{r}}=\overline{\mathbf{u}}_{0_{r}}, \quad r=1,2, \ldots ., 2 n
$$

where $\bar{\lambda}_{0}$ and $\overline{\mathbf{u}}_{0}$ are the complex conjugate of $\lambda_{0}$ and $\mathbf{u}_{0}$, respectively. Equation (18) indicates that the left eigenvectors are exactly the complex conjugates of the right eigenvectors. This is due to the nature of the unperturbed eigensystem in which $A_{0}$ is symmetric and $B_{0}$ is skew symmetric. Also, Eq. (18) simply states that there is no necessity to solve the unperturbed eigenvalue problem twice to have right and left eigenvectors because they are complex conjugates. Now consider the biorthogonality related Eqs (12) upon which the results of the general second order perturbation theory in the preceding section are derived. And consider an unperturbed eigenproblem of order $2 n=2$ having 2 eigenvalues, 2 right eigenvectors and 2 left eigenvectors. Taking into consideration that the unperturbed system is conservative, and upon using Eqs. (17) and (18), the following orthogonality conditions hold true:

$$
\begin{gathered}
\mathbf{u}_{01}^{T} A_{0} \mathbf{u}_{01}=2 a_{1} \delta_{11}, \quad \mathbf{u}_{01}^{T} A_{0} \mathbf{u}_{02}=0, \\
\mathbf{u}_{02}^{T} A_{0} \mathbf{u}_{01}=0, \mathbf{u}_{02}^{T} A_{0} \mathbf{u}_{02}=2 a_{2} \delta_{22} \\
\mathbf{v}_{01}^{T} A_{0} \mathbf{u}_{01}=\overline{\mathbf{u}}_{01}^{T} A_{0} \mathbf{u}_{01}=\mathbf{u}_{02}^{T} A_{0} \mathbf{u}_{01}=0, \\
\mathbf{v}_{01}^{T} A_{0} \mathbf{u}_{02}=\overline{\mathbf{u}}_{01}^{T} A_{0} \mathbf{u}_{02}=\mathbf{u}_{02}^{T} A_{0} \mathbf{u}_{02}=2 a_{2} \delta_{12} \\
\mathbf{v}_{02}^{T} A_{0} \mathbf{u}_{01}=\overline{\mathbf{u}}_{02}^{T} A_{0} \mathbf{u}_{01}=\mathbf{u}_{01}^{T} A_{0} \mathbf{u}_{01}=2 a_{1} \delta_{21} \\
\mathbf{v}_{02}^{T} A_{0} \mathbf{u}_{02}=\overline{\mathbf{u}}_{02}^{T} A_{0} \mathbf{u}_{02}=\mathbf{u}_{01}^{T} A_{0} \mathbf{u}_{02}=0
\end{gathered}
$$

If one considers the Kronecker product properties

$$
\delta_{i j}=\left\{\begin{array}{lll}
1 & \text { for } & i=j \\
0 & \text { for } & i \neq j
\end{array},\right.
$$

for application to Eqs. (19) and (20), it follows that the results of the biorthogonality multiplications in Eqs. (19), if arranged in a matrix form, lead to a diagonal matrix, while the multiplications in Eqs. (20) will lead to a matrix of zero elements. On the basis of this result, one can conclude that using the left eigenvector $v_{0}$ in the biorthogonality relations does not justify the arrangements of Eq. (12), and hence a mismatch occurs in the formulation of the second order perturbation theory leading to incorrect computations if the solution results (Eqs. (A4) through (A8)) are used in their current form. This proves the first part of the Theorem $I$. As a result to this, $-\mathbf{u}_{0}$ should replace $\mathbf{v}_{0}$ in the formulation starting with Eq. (13c), which will be modified to

$$
\mathbf{v}_{i}=\overline{\mathbf{u}}_{0 i}+\overline{\mathbf{u}}_{1 i}+\overline{\mathbf{u}}_{2 i}+\ldots
$$


Or in a more convenient form to the perturbation process:

$$
\begin{gathered}
\mathbf{v}_{i}=-\mathbf{u}_{0 i}-\widetilde{\mathbf{u}}_{1 i}-\widetilde{\mathbf{u}}_{2 i}-\ldots ., \quad r=1,2, \ldots ., 2 n \\
\widetilde{\mathbf{u}}_{1 i}=\sum_{k=1}^{2 n} \dot{\gamma}_{i k} \mathbf{u}_{0 k}, \quad \widetilde{\mathbf{u}}_{2 i}=\sum_{k=1}^{2 n} \widetilde{\gamma}_{i k} \mathbf{u}_{0 k}, \quad r=1,2, \ldots ., 2 n
\end{gathered}
$$

The assumption made to develop Eqs. (22) : (24) is mainly based on the nature of the unperturbed system (11) in which the left eigenvectors are the complex conjugates of the right eigenvectors. If one substitutes Eqs. (8), (13a), (13b) and (23) into Eq. (4) and then collects by the order of magnitudes, then three sets of problems of different perturbation orders result:

$$
\begin{aligned}
& \widetilde{\mathbf{O}}(0):-\mathbf{u}_{0 j}^{T} A_{0} \mathbf{u}_{0 i}=2 a_{i} \delta_{i j} \\
& -\mathbf{u}_{0 j}^{T} B_{0} \mathbf{u}_{0 i}=2 a_{i} \lambda_{0 i} \delta_{i j}, \quad i, j=1,2, \ldots ., 2 n \\
& \widetilde{\mathbf{O}}(1):-\mathbf{u}_{0 j}^{T} A_{0} \mathbf{u}_{1 i}-\mathbf{u}_{0 j}^{T} A_{1} \mathbf{u}_{0 i}-\widetilde{\mathbf{u}}_{1 j}^{T} A_{0} \mathbf{u}_{0 i}=0 \\
& -\mathbf{u}_{0 j}^{T} B_{0} \mathbf{u}_{1 i}-\mathbf{u}_{0 j}^{T} \boldsymbol{B}_{1} \mathbf{u}_{0 i}-\widetilde{\mathbf{u}}_{1 j}^{T} \boldsymbol{B}_{0} \mathbf{u}_{0 i}=2 a_{i} \lambda_{1 i} \delta_{i j}, i, j=1,2, \ldots, 2 n \\
& \widetilde{\mathbf{O}}(2):-\mathbf{u}_{0 j}^{T} A_{0} \mathbf{u}_{2 i}-\mathbf{u}_{0 j}^{T} A_{1} \mathbf{u}_{1 i}-\widetilde{\mathbf{u}}_{1 j}^{T} A_{0} \mathbf{u}_{1 i}-\widetilde{\mathbf{u}}_{1 j}^{T} A_{1} \mathbf{u}_{0 i}-\widetilde{\mathbf{u}}_{2 j}^{T} A_{0} \mathbf{u}_{0 i}=0 \\
& -\mathbf{u}_{0 j}^{T} \boldsymbol{B}_{0} \mathbf{u}_{2 i}-\mathbf{u}_{0 j}^{T} \boldsymbol{B}_{1} \mathbf{u}_{1 i}-\widetilde{\mathbf{u}}_{1 j}^{T} \boldsymbol{B}_{0} \mathbf{u}_{1 i}-\widetilde{\mathbf{u}}_{1 j}^{T} \boldsymbol{B}_{1} \mathbf{u}_{0 i}-\widetilde{\mathbf{u}}_{2 j}^{T} \boldsymbol{B}_{0} \mathbf{u}_{0 i}=2 a_{i} \lambda_{2 i} \delta_{i j} \\
& i, j=1,2, \ldots ., 2 n
\end{aligned}
$$

where $\widetilde{\mathbf{O}}(0), \widetilde{\mathbf{O}}(1)$ and $\widetilde{\mathbf{O}}(2)$ indicate the modified zero-, first- and secondorder perturbation problems, respectively. Substituting Eqs. (14a), (15a) and (24a) into (26), with orthogonality relations like (19) being utilized, the firstorder perturbation solution is provided as in Eqs (A4) and (A5) except that $-\mathbf{u}_{0 j}$ replaces $\mathbf{v}_{0 j}$ in these equations. Similarly, the substitution of Eqs. (14b), (15b) and (24b) into Eq. (27), and upon the use of orthogonality relations as in Eqs. (19), the second-order solution will be the same as that in Eqs. (A6) : (A8), except that the sign of all terms at the right hand sides of these equation is reversed. This completes the proof of theorem $I$.

\section{SIMPLIFIED UNPERTURBED CALCULATIONS}

Although a contribution is made to the second order perturbation theory in the preceding section, the vibration analyst is still in need to a powerful tool by which an unperturbed eigensolution can be systematically generated and a considerable save in time can be ultimately achieved for large scale systems. Once again, the special form of the unperturbed conservative gyroscopic system can be utilized. Meirovitch $[25,26]$ has shown that a conservative gyroscopic eigensystem like the one of Eq. (11) can be transformed into a highly standard 
eigenvalue problem of a single, real, positive definite symmetric matrix. The resulting eigenvalues and eigenvectors of this problem will be real. So many fast, efficient algorithms are available for solving the later problem. The procedure of transformation is as follows:

Consider the following unperturbed eigenvalue problem for a conservative gyroscopic system where the eigenvalues are normally pure imaginary:

$$
-B_{0} \mathrm{u}_{0}=\mathrm{i} \omega_{0} A_{0} \mathrm{u}_{0}, \quad A_{0}^{T}=A_{0} \succ 0, \quad B=-B^{T}
$$

The complex eigensolution of (28) can be expressed as:

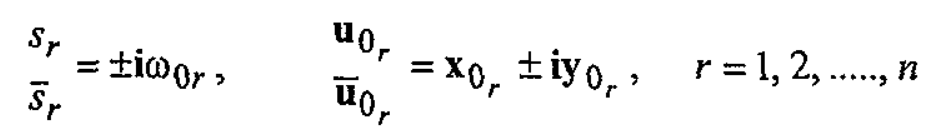

To transform the problem from complex to a real form $\mathbf{u}_{0}=\mathbf{x}_{0}+\mathbf{i y} \mathbf{y}_{0}$ is substituted into Eq. (28). Then both the real and imaginary part on both sides are equated to give

$$
-B_{0} \mathbf{x}_{0}=\omega_{0} A_{0} \mathbf{y}_{0},+B_{0} \mathbf{y}_{0}=\omega_{0} A_{0} \mathbf{x}_{0}
$$

Solving Eqs. (30a,b) together, provides

$$
B_{0}^{*} \mathbf{x}_{0}=\lambda_{0} A_{0} \mathbf{x}_{0}, \quad B_{0}^{*} \mathbf{y}_{0}=\lambda_{0} A_{0} \mathbf{y}_{0}, \quad \lambda_{0}=\omega_{0}^{2}
$$

where

$$
B_{0}^{*}=B_{0}^{T} A_{0}^{-1} B_{0}
$$

is a symmetric positive definite matrix. Since $A_{0}$ is symmetric positive it can be decomposed by Cholesky decomposition as follows:

$$
A_{0}=Q^{T} Q
$$

where $Q$ is a $2 n \times 2 n$ nonsingular, orthogonal matrix such that $Q^{-1}=Q^{T}$. By using the linear transformation

$$
Q \mathbf{x}=\mathbf{z}_{x}, \quad Q \mathbf{y}=\mathbf{z}_{y}
$$

the eigenvalue problem of equation (31) can be reduced to the following standard real one: 


$$
A_{0}^{*} \mathrm{z}=\lambda_{0} \mathrm{z}, \quad \lambda_{0}=\omega_{0}^{2}
$$

where the last two equations implicitly means that $\mathbf{z}_{x}$ is the same as $\mathbf{z}_{y}$, and

$$
A_{0}^{*}=\left(Q^{T}\right)^{-1} B_{0}^{*} Q^{-1}=\left(Q^{-1}\right)^{T} B_{0}^{*} Q^{-1},
$$

The two eigenvalue problems (31) and (35) have the same eigenvalues with each eigenvalue of $A_{0}^{*}$ retains the multiplicity of two. This multiplicity is expressed as in Eq. (16). By analogy with Eq. (34), the real and imaginary part of $\mathbf{u}_{0_{r}}$ can be expressed as follows:

$$
\mathbf{x}_{0, r}=Q^{-1} \mathbf{z}_{2 r-1}, \quad \mathbf{y}_{0 r}=Q^{-1} \mathbf{z}_{2 r}, \quad r=1,2, \ldots ., n
$$

Thus the complex eigensolution of Eq. (29), can be reconstructed from the solution of a highly standard eigenvalue problem of single, symmetric positive definite matrix with real eigenvalues and eigenvectors. This, of course, leads to a marginal reduction in the computational time. This reduction becomes more effective as the order of the problem increases. Another idea for future work is that the first and second order perturbation solutions in equations (A4) through (A8) can be related directly to the calculated real eigenvectors (37) rather than reconstructing the complex eigenvector from these real ones. This will save a great part in core of the computer used.

\section{RESULTS AND DISCUSSIONS}

A little problem is to be highlighted first. A hard condition has to be met by any eigenvalue problem in order to be solved by the perturbation method developed in this paper. The entries of the perturbation matrices $\boldsymbol{A}_{1}$ and $\boldsymbol{B}_{1}$ should be one order of magnitude smaller than the entries of the unperturbed matrices $\boldsymbol{A}_{0}$ and $\boldsymbol{B}_{0}$. This implicitly means, according to Eqs. (9) and (10) that the entries of the symmetric damping matrix $C_{0}$ should be one order of magnitude smaller than the entries of the skew symmetric damping matrix $\mathrm{Cg}$. The situation is hard to meet for some applications. Consequently, the theory will not be applicable for those applications. To avoid such a situation, the symmetric damping matrix $C_{0}$ can be divided as follows:

$$
C_{0}=C_{0 \mathrm{p}}+C_{0 \mathrm{np}}
$$

where $C_{0 \mathrm{p}}$ is the part of the symmetric damping matrix $C_{0}$ that is proportional to the distribution of the mass and stiffness matrices such that: 


$$
C_{0_{\mathrm{p}}}=\alpha M_{\mathrm{o}}+\beta K_{\mathrm{o}}
$$

where $\alpha$ and $\beta$ are proportionality constants. $C_{0_{\mathrm{np}}}$ is the nonproportional part that will replace $C_{0}$ in Eq. (10) such that the modified formulation matrices will be:

$$
\begin{gathered}
A_{0}=\left[\begin{array}{cc}
K_{0} & 0 \\
0 & M_{0}
\end{array}\right], \quad B_{0}=\left[\begin{array}{cc}
0 & -K_{0} \\
K_{0} & G+C_{0 \mathrm{p}}+C_{\mathrm{g}}
\end{array}\right] \\
A_{1}=\left[\begin{array}{cc}
-K_{\mathrm{g}}^{\mathrm{T}}-H^{\mathrm{T}} & 0 \\
0 & M_{\mathrm{g}}
\end{array}\right], B_{1}=\left[\begin{array}{cc}
0 & K_{\mathrm{g}}^{\mathrm{T}}+H^{\mathrm{T}} \\
K_{\mathrm{g}}+H & C_{0 \mathrm{np}}
\end{array}\right]
\end{gathered}
$$

Or, equivalently, the last two equations can be rewritten as follows:

$$
\begin{gathered}
A_{0}=\left[\begin{array}{cc}
K_{0} & 0 \\
0 & M_{0}
\end{array}\right], \quad B_{0}=\left[\begin{array}{cc}
0 & -K_{0} \\
K_{0} & G+C_{0 \mathrm{p}}
\end{array}\right] \\
A_{1}=\left[\begin{array}{cc}
-K_{\mathrm{g}}^{\mathrm{T}}-H^{\mathrm{T}} & 0 \\
C_{\mathrm{g}} & M_{\mathrm{g}}
\end{array}\right], \quad B_{1}=\left[\begin{array}{cc}
0 & \boldsymbol{K}_{\mathrm{g}}^{\mathrm{T}}+\boldsymbol{H}^{\mathrm{T}} \\
\boldsymbol{K}_{\mathrm{g}}+\boldsymbol{H} & C_{0_{\mathrm{np}}}
\end{array}\right]
\end{gathered}
$$

With these alternating arrangement in the modified formulation matrices of Eqs. (40) : (43), most of the nonclassically damped systems that do not meet the order of magnitude condition can be solved by the perturbation technique developed in this paper.

\section{Example1:}

The following hypothetical 2-DOF system is used to show the accuracy of solutions obtained by the current method in comparison with those obtained by exact methods. This example slightly violates the condition that the entries of $A_{1}$ and $B_{1}$ are one order of magnitude smaller than the entries of the matrices $\boldsymbol{A}_{0}$ and $\boldsymbol{B}_{0}$. This is just to show that the method presented here is capable of producing acceptable solutions even when this hard condition is violated. The matrices in Eq. (1) are given by:

$$
\boldsymbol{M}=\left[\begin{array}{cc}
5 & 2 \\
3 & 6.5
\end{array}\right], \quad \boldsymbol{C}=\left[\begin{array}{cc}
1 & 0.5 \\
0.3 & 1
\end{array}\right], \quad \boldsymbol{G}=\left[\begin{array}{cc}
0 & -4 \\
+4 & 0
\end{array}\right], \quad K=\left[\begin{array}{ll}
4 & 0 \\
0 & 5
\end{array}\right]
$$




$$
\boldsymbol{H}=\left[\begin{array}{cc}
0 & -1 \\
+1 & 0
\end{array}\right]
$$

According to Eqs. (6) and (7), the resulting $n \times n$ formulation matrices are :

$$
\begin{gathered}
M_{0}=\left[\begin{array}{cc}
5 & 2.5 \\
2.5 & 6.5
\end{array}\right], \quad M_{g}=\left[\begin{array}{cc}
0 & -0.5 \\
+0.5 & 0
\end{array}\right], \quad C_{0}=\left[\begin{array}{cc}
1 & 0.4 \\
0.4 & 1
\end{array}\right], \\
C_{g}=\left[\begin{array}{cc}
0 & +0.1 \\
-0.1 & 0
\end{array}\right], \quad K_{0}=\left[\begin{array}{ll}
4 & 0 \\
0 & 5
\end{array}\right], \quad K_{g}=\left[\begin{array}{ll}
0 & 0 \\
0 & 0
\end{array}\right],
\end{gathered}
$$

The $2 n \times 2 n$ formulation matrices in Eqs. (8), (9) and (10) are then given by:

$$
\begin{aligned}
& A_{0}=\left[\begin{array}{cccc}
4 & 0 & 0 & 0 \\
0 & 5 & 0 & 0 \\
0 & 0 & 5 & 2.5 \\
0 & 0 & 2.5 & 6.5
\end{array}\right], B_{0}=\left[\begin{array}{cccc}
0 & 0 & -4 & 0 \\
0 & 0 & 0 & -5 \\
+4 & 0 & 0 & -3.9 \\
0 & +5 & +3.9 & 0
\end{array}\right] \\
& A_{1}=\left[\begin{array}{cccc}
0 & -1 & 0 & 0 \\
+1 & 0 & 0 & 0 \\
0 & 0 & 0 & -0.5 \\
0 & 0 & +0.5 & 0
\end{array}\right], B_{1}=\left[\begin{array}{cccc}
0 & 0 & 0 & +1 \\
0 & 0 & -1 & 0 \\
0 & -1 & 1 & 0.4 \\
+1 & 0 & 0.4 & 1
\end{array}\right]
\end{aligned}
$$

Comparing the entries of the matrices $A_{1}$ and $B_{1}$ with those of the matrices $A_{0}$ and $B_{0}$ one notices that they violate the condition as mentioned above. The solution results are shown in Table 1 . The computed damping ratios for the two modes are actually contained into the symmetric damping matrix $C_{0}$, and are given by $\zeta_{1}=0.086$ and $\zeta_{2}=0.207$, respectively. The results show a significant matching between the second order perturbation results and those obtained by exact methods. Accuracy to the third decimal is achieved by the current method when compared to the exact one even with the order of magnitude condition is violated.

Table 1. Eigenvalues obtained by perturbation and by exact methods.

\begin{tabular}{|c|c|c|c|}
\hline Exact solution & $\widetilde{\mathbf{O}}(0)$ & $\widetilde{\mathbf{O}}(0)+\widetilde{\mathbf{O}}(1)$ & $\widetilde{\mathbf{O}}(0)+\widetilde{\mathbf{O}}(1)+\widetilde{\mathbf{O}}(2)$ \\
\hline$-0.1262 \pm 1.4548$ & $\pm 0.1287 \mathbf{i}$ & $-0.1287 \pm 1.4736 \mathbf{i}$ & $-0.1287 \pm 1.4545 \mathbf{i}$ \\
\hline$-0.1267 \pm 0.5963$ & $\pm 0.1266 \mathbf{i}$ & $-0.1266 \pm 0.5924 \mathbf{i}$ & $-0.1266 \pm 0.5962 \mathbf{i}$ \\
\hline
\end{tabular}




\section{Example2:}

This example shows how to handle nonproportionally damped systems in cases where the matrix $C_{0}$ does not justify the order of magnitude condition. The matrices in Eq. (1) are given by:

$$
\begin{gathered}
M=\left[\begin{array}{ll}
3 & 0 \\
0 & 4
\end{array}\right], \quad \boldsymbol{C}=\left[\begin{array}{cc}
3 & -1.4 \\
1.6 & 4
\end{array}\right], \quad \boldsymbol{G}=\left[\begin{array}{cc}
0 & -4 \\
+4 & 0
\end{array}\right], \quad K=\left[\begin{array}{ll}
5 & 0 \\
0 & 7
\end{array}\right] \\
H=\left[\begin{array}{cc}
0 & -0.5 \\
+0.5 & 0
\end{array}\right]
\end{gathered}
$$

According to Eqs. (6) and (7), the resulting $n \times n$ formulation matrices are :

$$
\begin{gathered}
M_{0}=\left[\begin{array}{ll}
3 & 0 \\
0 & 4
\end{array}\right], \quad M_{\mathrm{g}}=\left[\begin{array}{ll}
0 & 0 \\
0 & 0
\end{array}\right], \quad C_{0}=\left[\begin{array}{cc}
3 & 0.1 \\
0.1 & 4
\end{array}\right], \\
C_{\mathrm{g}}=\left[\begin{array}{cc}
0 & -1.5 \\
1.5 & 0
\end{array}\right], \quad K_{0}=\left[\begin{array}{ll}
5 & 0 \\
0 & 7
\end{array}\right], \quad K_{\mathrm{g}}=\left[\begin{array}{ll}
0 & 0 \\
0 & 0
\end{array}\right],
\end{gathered}
$$

According to Eq. (39), with proportionality constants $\alpha=\beta=0.35$, the proportional and nonproportional parts of the matrix $C_{0}$ are given by:

$$
C_{0 \mathrm{p}}=\left[\begin{array}{cc}
2.8 & 0 \\
0 & 3.85
\end{array}\right], \quad C_{0 \mathrm{np}}=\left[\begin{array}{cc}
0.2 & 0.1 \\
0.1 & 0.15
\end{array}\right] \text {, }
$$

The $2 n \times 2 n$ formulation matrices in Eqs. (8), (9) and (10) are then given by:

$$
\begin{gathered}
A_{0}=\left[\begin{array}{llll}
5 & 0 & 0 & 0 \\
0 & 7 & 0 & 0 \\
0 & 0 & 3 & 0 \\
0 & 0 & 0 & 4
\end{array}\right], B_{0}=\left[\begin{array}{cccc}
0 & 0 & -5 & 0 \\
0 & 0 & 0 & -7 \\
+4 & 0 & 2.8 & -5.5 \\
0 & +7 & +5.5 & 3.85
\end{array}\right] \\
A_{1}=\left[\begin{array}{cccc}
0 & -0.5 & 0 & 0 \\
+0.5 & 0 & 0 & 0 \\
0 & 0 & 0 & 0 \\
0 & 0 & 0 & 0
\end{array}\right], B_{1}=\left[\begin{array}{cccc}
0 & 0 & 0 & +0.5 \\
0 & 0 & -0.5 & 0 \\
0 & -0.5 & 0.2 & 0.1 \\
+0.5 & 0 & 0.1 & 0.15
\end{array}\right]
\end{gathered}
$$


The calculated damping ratios of the two modes are $\zeta_{1}=0.38$ and $\zeta_{2}=0.30$, respectively. The results are shown in Table 2 . The accuracy achieved in this example is also considerable.

Table 2 . Eigenvalues obtained by perturbation and by exact methods.

\begin{tabular}{|c|c|c|c|}
\hline Exact solution & $\widetilde{\mathbf{O}}(0)$ & $\widetilde{\mathbf{O}}(0)+\widetilde{\mathbf{O}}(1)$ & $\widetilde{\mathbf{O}}(0)+\widetilde{\mathbf{O}}(1)+\widetilde{\mathbf{O}}(2)$ \\
\hline$-0.7220 \pm 2.2559 \mathbf{i}$ & $\pm 2.2842 \mathbf{i}$ & $-0.7244 \pm 2.2842 \mathbf{i}$ & $-0.7248 \pm 2.2840 \mathbf{i}$ \\
\hline$-0.2780 \pm 0.6681 \mathbf{i}$ & $\pm 0.6678 \mathbf{i}$ & $-0.2755 \pm 6648 \mathbf{i}$ & $-0.2775 \pm 0.6681 \mathbf{i}$ \\
\hline
\end{tabular}

\section{Example3:}

This example is devoted to a practical problem. A rotor shaft, supported on two identical, tilting 5-pad bearings with the bearing load acting between pads, is considered in this example as shown in Fig. 1. The bearing data are such that the preload factor is taken 0.66 , the length is $0.025 \mathrm{~m}$, the diameter is $0.05 \mathrm{~m}$, the raciial clearance is $0.001 \mathrm{~m}$, the lubricant viscosity is $0.069 \mathrm{~N} . \mathrm{s} / \mathrm{m}$. The bearing stiffness and damping coefficients are then taken by interpolation from the tabulated coefficients by Someya [27]. The disk mass (per bearing) 150.03 $\mathrm{kg}$, the journal mass is $141.47 \mathrm{~kg}$, the bearing-support mass is $100.8 \mathrm{~kg}$. The rotor stiffness is $49 \times 10^{6} \mathrm{~N} / \mathrm{m}$ and the support stiffness is $10 \times 10^{7}$. Damping is neglected in both the rotor and the support. The 6-DOF model considered here has been frequently used for studying the lateral vibration of rotors in two perpendicular $\mathrm{x}$ and $\mathrm{y}$ directions as shown in Fig. 1. The model equations of motion are reported by Abduljabbar et al. [28]. The rotor speed is considered to be $1230 \mathrm{rad} / \mathrm{s}$. The results are shown in Table 3 where the perturbation approach developed in this paper is still holding a reasonable accuracy in comparison with the exact method.

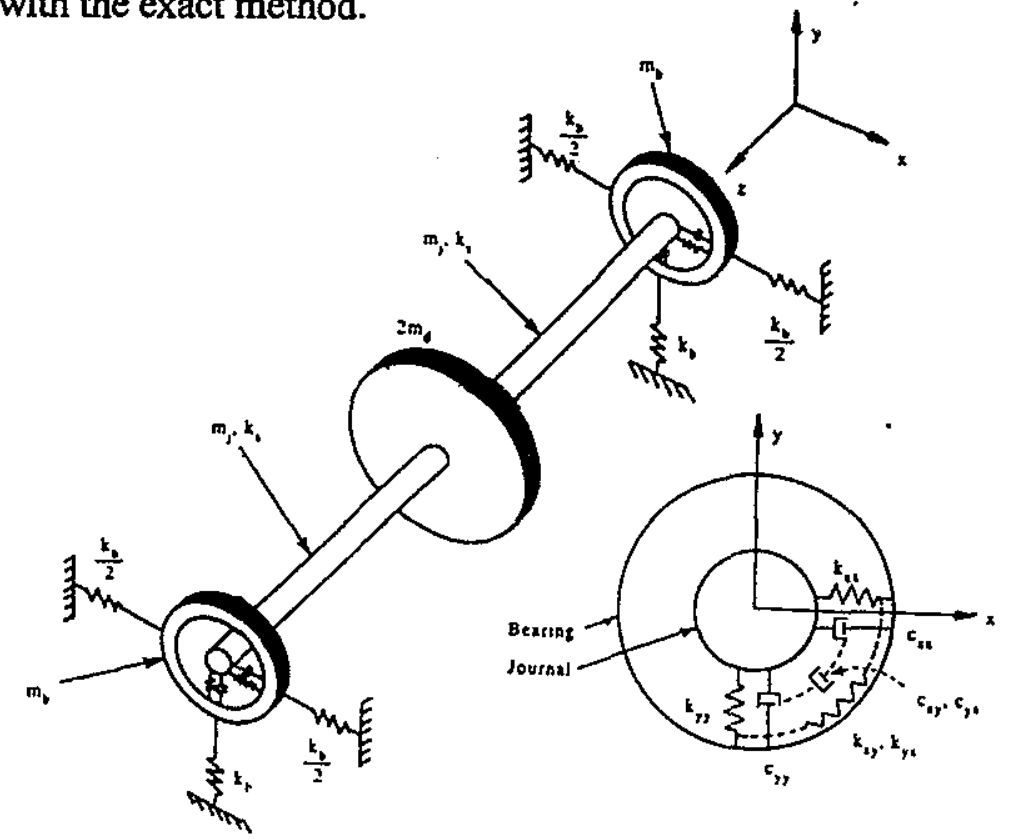

Fig. 1 Rotor-bearing systeñi model (after Abduliabbar et. Al. [28]). 
Table 3. Eigenvalues obtained by perturbation and by exact methods ${ }^{*}$

\begin{tabular}{|c|c|c|c|}
\hline Exact solution & $\widetilde{\mathbf{O}}(0)$ & $\widetilde{\mathbf{O}}(0)+\widetilde{\mathbf{O}}(1)$ & $\widetilde{\mathbf{O}}(0)+\widetilde{\mathbf{O}}(1)+\widetilde{\mathbf{O}}(2)$ \\
\hline$-0.0004 \pm 0.1441 \mathbf{i}$ & $\pm 0.1440 \mathbf{i}$ & $-0.0004 \pm 0.1440 \mathbf{i}$ & $-0.0004 \pm 0.1441 \mathbf{i}$ \\
\hline$-0.0003 \pm 0.1496 \mathbf{i}$ & $\pm 0.1496 \mathbf{i}$ & $-0.0003 \pm 0.1496 \mathbf{i}$ & $-0.0003 \pm 0.1496 \mathbf{i}$ \\
\hline$-0.0211 \pm 0.6182 \mathbf{i}$ & $\pm 0.6159 \mathbf{i}$ & $-0.0211 \pm 0.6159 \mathbf{i}$ & $-0.0213 \pm 0.6183 \mathbf{i}$ \\
\hline$-0.0118 \pm 0.6815 \mathbf{i}$ & $\pm 0.6789 \mathbf{i}$ & $-0.0121 \pm 0.6789 \mathbf{i}$ & $-0.0124 \pm 0.6811 \mathbf{i}$ \\
\hline$-0.0485 \pm 0.9460 \mathbf{i}$ & $\pm 0.9500 \mathbf{i}$ & $-0.0485 \pm 0.9500 \mathbf{i}$ & $-0.0483 \pm 0.9462 \mathbf{i}$ \\
\hline$-0.1175 \pm 1.1264 \mathbf{i}$ & $\pm 1.1345 \mathbf{i}$ & $-0.1172 \pm 1.1345 \mathbf{i}$ & $-0.1169 \pm 1.1294 \mathbf{i}$ \\
\hline
\end{tabular}

* All numbers in the table should be multiplied by $10^{3}$

\section{CONCLUSIONS}

A method is developed to get the general second-order perturbation theory fruitfully applicable to the solution of the eigenvalue problem of nonclassically, viscously damped system. The main contribution here is that the eigensolution of a highly standard eigenvalue problem of single, symmetric positive definite matrix is systematically employed to generate the eigensolution of an asymmetric non-proportionally damped eigenproblem. The later one primarily includes asymmetric damping, stiffness and mass matrices introduced by gyroscopic and circulatory effects. A high compatibility between solutions obtained by the current method and those obtained by exact method is justified.

\section{REFERENCES}

1. Caughey, T. K. and O'Kelly, M. E. J., "Classical normal modes in damped linear dynamic systems", ASME Trans., J. Applied Mechanics, V:ol. 32, pp $583-588,1965$

2. Meirovitch, L., "Principles and techniques of vibrations", Chapts. 4-6, Prentice-Hall, Inc., 1997

3. Udwadia, F. E. and Esfandiari, R. S., "Nonclassically damped dynamic systems: An iterative approach" ASME Trans., J. Applied mechanics, Vol. 57, pp 423-433, 1990.

4. Cronin, D. L., "Approximation for determining harmonically excited response of nonclassically damped systems" ASME Trans., J. Engineering for Industry, pp 43-47, 1976.

5. Hwang, J. H. and Ma, F., "On the approximate solution of nonclassically damped linear systems", ASME Trans., J. Applied Mechanics, Vol. 60, pp 695-701, 1993.

6. Gupta, K. K., "Eigenproblem solution of damped structural systems", Int. J. Numer. Meth. Engng, Vol. 8, pp 877-911, 1974.

7. ElBeheiry, E. M., "A method for preview vibration control of systems having forcing inputs and rapidly switched dampers", J. Sound \& Vibration, Vol. 213, No. 2, pp 269-283, 1998. 
8. ElBeheiry, E. M., "Suboptimal bilinear control methods applied to suppressing car vibrations", J. Vibration \& Control, Vol. 7, pp 279-306, 2001.

9. Brandon, J. A., "A discussion of alternative Duncan formulations of the solution of nonclassically, viscously damped linear systems", ASME Trans., J. Applied mechanics, Vol. 51, pp 904-906, 1984.

10. Fawzy, I. And Bishop, R. E. D., "On the dynamics of linear nonconservative systems" Proceedings of the Royal Society, London, Series A, Vol. 352, pp 25-40, 1976.

11. Washed, I. F. A. And Bishop, R.E.D., "On the equations governing the free and forced vibrations of a general non-conservative system" J. Mechanical Engineering Science, Vol. 18, No. 1, pp 6-10, 1976.

12. Newland, D. E., "On the modal analysis of non-conservative linear systems" J. Sound \& Vibration, Vol. 112, No. 1, pp 69-96, 1987.

13. Munjiza, A., Owen, D. R. J. and Crook, A. J. L., "An $\mathbf{M}\left(\mathbf{M}^{-1} \mathbf{K}\right)^{\mathrm{m}}$ proportional damping in explicit integration of dynamic structural systems" int. J. Numer. Meth. Engng., Vol. 41, pp 1277-1296, 1998.

14. Inman, D. J., "Dynamics of asymmetric nonconservative systems", ASME Trans., J. Applied mechanics, Vol. 50, pp 199-204, 1983.

15. Ahmadian, M. and Inman, D. J., "Classical normal modes in asymmetric nonconservative dynamic systems" AIAA Journal, Vol. 22, No. 7, pp 1012$1015,1983$.

16. Ahmadian, M. and Inman, D. J., "On the nature of eigenvalues of general nonconservative systems" ASME Trans., J. Applied mechanics, Vol, 51, pp 193-194, 1984.

17. Fox, R. L. and Kapoor, M. P., "Rates of changes of eigenvalues and eigenvectors" AIAA Journal, Vol. 6, No. 12, pp 2426-2429, 1968.

18. Plaut, R. H. and Huseyin, K., "Derivatives of eigenvalues and eigenvectors in non-self-adjoint systems", AIAA Journal, Vol. 11, No. 2, pp 250-251, 1973.

19. Meirovitch, L., "Computational methods in structural dynamics", Sijthoff and Noordhoff, Netherlands, 1980.

20. Bickford, W., "An improved computational technique for perturbations of the generalized symmetric linear algebraic eigenvalue problem" Int. J. Numer. Meth. Engng., Vol. 24, pp 529-541, 1987.

21. Meirovitch, L. and Ryland. II, G., "Response of slightly damped gyroscopic systems" J. Sound \& Vibration, Vol. 67, No. 1, pp'1-19, 1979.

22. Chung, K. R. and Lee, C. W., "Dynamic reanalysis of weakly nonproportionally damped systems" Journal of Sound and Vibration, Vol. 111, No. 1, pp 37-50, 1986.

23. Genta, G., "Whirling of unsymmetric rotors: A finite element approach based on complex co-ordinates", J. Sound \& Vibration, Vol. 124, No. 1, pp 27-53, 1988.

24. Holmes, R., "Vibration. of rotor-bearing assemblies", Proc. NATO Advanced Institute on Vibration and Wear Damage in High Speed Rotating 
Machinery, Troia, Sebutal, Portogal, April 10-22, Kluwer Academic Publisher, The Netherland, pp 279-297, 1990.

25. Meirovitch, L., "A new method of solution of the eigenvalue problem for gyroscopic systems", AIAA J., Vol. 12, No. 10, pp 1337-1342, 1974.

26. Meirovitch, L., "A modal analysis for the response of linear gyroscopic systems", ASME Trans., J. Applied mechanics, Vol. 42, pp 446-450, 1975.

27. Someya, T. (editor), "Journal-bearing data book", Springer-Verlag Berlin, Heidlberg, Germany, 1989.

28. Abduljabbar, Z., ElMadany, M. M., and Al-Bahkali, E., "On the vibration and control of a flexible rotor mounted on fluid film bearing", Computers and Structures, Vol. 65, No. 6, pp 849-856, 1997.

\section{APPENDIX A: GENERAL PERTURBATION RESULTS}

The perturbation results according to the order of magnitude as derived by Chung and Lee [20] are:

$$
\begin{aligned}
& \mathbf{O}(0): \mathbf{v}_{0 j}^{T} A_{0} \mathbf{u}_{0 i}=2 a_{i} \delta_{i j} \\
& \mathbf{v}_{0 j}^{T} \boldsymbol{B}_{0} \mathbf{u}_{0 i}=2 a_{i} \lambda_{0 i} \delta_{i j}, \quad i, j=1,2, \ldots, 2 n \\
& \mathbf{O}(1): \mathbf{v}_{0 j}^{T} A_{0} \mathbf{u}_{1 i}+\mathbf{v}_{0 j}^{T} A_{1} \mathbf{u}_{0 i}+\mathbf{v}_{1 j}^{T} A_{0} \mathbf{u}_{0 i}=0 \\
& \mathbf{v}_{0 j}^{T} B_{0} \mathbf{u}_{1 i}+\mathbf{v}_{0 j}^{T} B_{1} \mathbf{u}_{0 i}+\mathbf{v}_{1 j}^{T} B_{0} \mathbf{u}_{0 i}=2 a_{i} \lambda_{1 i} \delta_{i j}, \quad i, j=1,2, \ldots ., 2 n \\
& \mathbf{O}(2): \mathbf{v}_{0 j}^{T} A_{0} \mathbf{u}_{2 i}+\mathbf{v}_{o j}^{T} A_{1} \mathbf{u}_{1 i}+\mathbf{v}_{1 j}^{T} A_{0} \mathbf{u}_{1 i}+\mathbf{v}_{1 j}^{T} A_{1} \mathbf{u}_{0 i}+\mathbf{v}_{2 j}^{T} A_{0} \mathbf{u}_{0 i}=0 \\
& \mathbf{v}_{0 j}^{T} B_{0} \mathbf{u}_{2 i}+\mathbf{v}_{o j}^{T} B_{1} \mathbf{u}_{1 i}+\mathbf{v}_{1 j}^{T} B_{0} \mathbf{u}_{1 i}+\mathbf{v}_{1 j}^{T} B_{1} \mathbf{u}_{0 i}+\mathbf{v}_{2 j}^{T} B_{0} \mathbf{u}_{0 i}=2 a_{i} \lambda_{2 i} \delta_{i j} \\
& i, j=1,2, \ldots ., 2 n
\end{aligned}
$$

where $\mathbf{O}(0), \mathbf{O}(1)$ and $\mathbf{O}(2)$ indicate the zero-, first- and second-order perturbation problems, respectively. Note here that Eqs. (A1) are similar to Eq. (12) of the eigenvalue problem for the unperturbed system. Substituting equations (13) into equation (A2), and upon using equations (11) and (A1), one gets the first order perturbation solutions:

$$
\begin{aligned}
& a_{j} \varepsilon_{i j}=\mathbf{v}_{0 j}^{T}\left(B_{1}-\lambda_{0 i} A_{1}\right) \mathbf{u}_{0 i} / 2\left(\lambda_{0 i}-\lambda_{0 j}\right) \\
& \begin{array}{l}
a_{i} \gamma_{i j}=\mathbf{v}_{0 j}^{T}\left(B_{1}-\lambda_{0 i} A_{1}\right) \mathbf{u}_{0 i} / 2\left(\lambda_{0 j}-\lambda_{0 i}\right), \\
i \neq j, \quad i, j=1,2, \ldots ., 2 n
\end{array} \\
& \begin{array}{l}
\varepsilon_{i i}=\gamma_{i i}=-\mathbf{v}_{0 i}^{T} A_{1} \mathbf{u}_{0 i} / 4 a_{i} \\
\lambda_{1 i}=\mathbf{v}_{0 i}^{T}\left(B_{1}-\lambda_{0 i} A_{1}\right) \mathbf{u}_{0 i} / 2 a_{i}, \quad i=j, \quad i=1,2, \ldots, 2 n
\end{array}
\end{aligned}
$$


Substituting equations (14) into equation (A3), and upon using equations (11), (A4) and (A5), one can extract the second order perturbation solutions. When $i \neq j$,

$$
\begin{gathered}
a_{j} \tilde{\varepsilon}_{i j}=\frac{1}{\left(\lambda_{0 i}-\lambda_{0 j}\right)}\left[-a_{i} \lambda_{1 i} \gamma_{j i}-a_{j} \lambda_{1 j} \varepsilon_{i j}+\sum_{k=1}^{2 n} a_{k} \gamma_{j k} \varepsilon_{i k}\left(\lambda_{0 i}-\lambda_{0 k}\right)\right]+ \\
\sum_{k=1}^{2 n} \varepsilon_{i k} \varepsilon_{k j} \\
a_{j} \tilde{\varepsilon}_{i j}=\frac{1}{\left(\lambda_{0 j}-\lambda_{0 i}\right)}\left[-a_{i} \lambda_{1 i} \gamma_{j i}-a_{j} \lambda_{1 j} \varepsilon_{i j}+\sum_{k=1}^{2 n} a_{k} \gamma_{j k} \varepsilon_{i k}\left(\lambda_{0 j}-\lambda_{0 k}\right)\right]+ \\
\sum_{k=1}^{2 n} \gamma_{j k} \gamma_{k i}
\end{gathered}
$$

When $i=j$,

$$
\begin{aligned}
& \tilde{\varepsilon}_{i i}=\tilde{\gamma}_{i i}=0.5 \sum_{k=1}^{2 n}\left[\gamma_{i k} \gamma_{k i}+\varepsilon_{i k} \varepsilon_{k i}+a_{k} \gamma_{i k} \varepsilon_{i k} / a_{i}\right] \\
& \lambda_{2 i}=\lambda_{1 i}\left(\gamma_{i i}+\varepsilon_{i i}\right)+\sum_{k=1}^{2 n} a_{k} \gamma_{i k} \varepsilon_{i k}\left(\lambda_{0 i}-\lambda_{0 k}\right) / a_{i}, \quad i=1,2, \ldots ., 2 n
\end{aligned}
$$


الحل المميز لمنظومات الإهتزاز الغير متماثلة والغيرتقليدية الإخماد

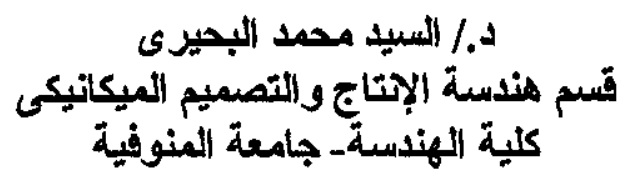

هذا البحث يقدم طريقة جديدة بإستخام التشويش الرياضى لإيجاد الحلول المميزة

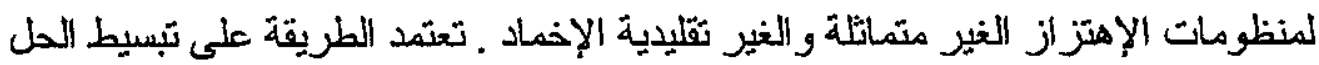

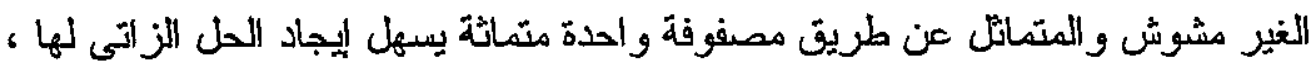
ثم بعد ذلك يتم إيجاد الحل الزاتى المشوش عن طريق الحل الغير المشوش بإستخدام نظرية تشويش من الدرجة الثانية تم تحقيقها رياضيا ونم أيضا تطبيقها على مثالين رقميين ومنظومة دوار يرتكز على محامل هيدروديناميكية ووجدت الحلول الناتجة من الطريقة

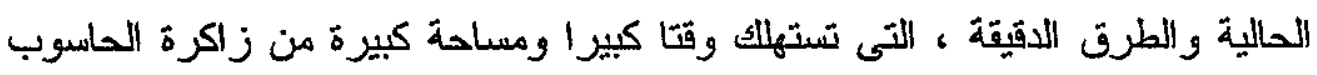
المستخدم ، متطابقة إلى حد كبير . 